within primary care could have occurred in ways other than our extra-mural service, but however such developments come about they do seem necessary for primary care to become an effective site for mental health promotion.

\section{Acknowledgements}

The funding for this project came from the Merton, Sutton and Wandsworth Health Authority's Specialist Health Promotion Service, for whose support we are grateful. We thank Dr Richard Chegwidden for his initial support for the project, and Dr Vino Frances, Dr Mary-Ellen White, Ms Julie Belfield, Ms Saira Razzaq and Ms Michelle Brown for their contribution to the project.

\section{References}

BROWN, M., GILLEARD, C. \& LOBO, R. (1997) Health In Mind. A Mental Health Promotion Service in Primary Care.
Evaluation Report. London: Merton. Sutton and Wandsworth Health Authority. Department of Public Health.

Hodgson, R. \& ABbassi, T. (1995) Effective Mental Health Premonition: A Review of the Effectiveness of Health Education and Health Promotion. Technical report No. 13. Cardiff: Health Promotion Wales.

TILFORD. S., DELANEY, F. \& VoGels, M. (1997) Effectiveness of Mental Health Promotion Interventions: A Review. London: Health Education Authority.

TUDOR-SMrTH, C. (1997) Commentary. In Effectiveness of Mental Health Promotion Interventions: A Review (eds S. Tulford. F. Delaney \& M. Vogels). London: Health Education Authority.

*Chris Gilleard, Director of Psychology. Pathfinder Mental Health Services, NHS Trust, Springfield University Hospital, Tooting, London SW17 7DJ; and Ros Lobo, Mental Health Primary Care Facilitator, Merton Sutton and Wandsworth Health Authority Specialist Health Promotion Service, Mitcham. Surrey

*Correspondence

\title{
Nature and extent of dental pathology and complications arising in patients receiving ECT
}

\author{
Nita Beli and Peter Bentham
}

\begin{abstract}
This study aimed to describe the prevalence of dental pathology in patients recelving electroconvulstve therapy and to prospectively determine the incidence of dental complications arising during treatment. Of 30 subjects, $93 \%$ complained of a dry mouth and $83 \%$ were taking drugs with onticholinergic properties. A third wore dentures and the dentate population had a mean of 15 decayed, miseing or filled teeth. Oral hygiene and periodontal condition was poor with one-third requiring scalling and $30 \%$ complex periodontal treatment. Temporomandibular pain followed $\mathbf{4 4 \%}$ of treatiments, and minor buccal lesions occurred in 22\%. Greater emphasis must be placed on dental care, and guidelines are suggested to improve practice.
\end{abstract}

Injuries to teeth and oral soft tissues are well known risks associated with electroconvulsive therapy (ECT). The muscles of mastication are directly depolarised by the electrical stimulus bypassing the effect of the muscle relaxant. High stresses are produced during the forceful closure of the jaws. The incisors are particularly at risk as they are normally inclined forwards. Strong occlusal forces will tend to rotate them on a transverse axis, the risk of damage being greater if malocclusions such as deep overbites, proclinations or retroclinations are present (Durrant, 1966). Uneven load distribution may result in the fracture or loosening of posterior 
teeth which if aspirated may cause serious complications.

Soft tissue lesions arise when the tongue, cheeks and lips get caught between the teeth or between the teeth and the mouthguard. Unopposed teeth may lacerate the gums in the opposite arch.

In periodontal disease there is gradual loss of connective tissue and resorption of the alveolar bone leading to loosening of the teeth. Caries can undermine the enamel of a crown or erode the neck of a tooth increasing the risk of fracture. Full dentures are usually removed prior to treatment but the situation regarding partial dentures and fixed prostheses is more complex.

An oral protective device is recommended in the routine practice of ECT with the aim of optimally distributing the occlusal load and protecting soft tissue from injury. The use of these devices in oral protection is well reviewed by Minneman (1995).

The American Psychiatric Association liability insurance programme receives few claims in relation to ECT, however a significant proportion of these are for dental injury (Slawson, 1989). Despite the clear importance of dental issues, little practical advice is given in The ECT Handbook (Royal College of Psychiatrists, 1995).

The aims of this study were to determine the prevalence and nature of dental disease in patients receiving ECT and to prospectively determine the incidence of oral soft tissue and dental complications arising during treatment. The findings, together with information from the literature, are utilised to formulate guidelines on dental management for ECT.

\section{The study}

The study population included all patients receiving ECT during a four-week period and all patients who had undergone treatment during their current admission to the Queen Elizabeth Psychiatric Hospital in Birmingham. A structured interview and dental examination was conducted. Subjects were asked about: oral hygiene habits; frequency of visits to the dentist: oral, dental and jaw problems together with a history of relevant physical illnesses and drug treatment. The case notes were examined and an ICD-10 (World Health Organization, 1992) diagnosis allocated. All subjects had an extraoral assessment of the neck, mandible and temporomandibular joint. Intra-orally soft tissues were assessed together with the number of intact, decayed, missing and filled teeth. Mobile and broken teeth were identified and plaque deposits recorded using a plaque index. The peridontal condition of each sextant was assessed utilising a basic peridontal examination (BPE) probe (Ainamo et al, 1982). The presence and condition of removable and fixed prostheses was recorded. Particular attention was paid to the presence of increased overbites, severe proclinations and retroclinations. Patients were observed during ECT for abnormal sounds and bleeding. The post-ECT examination was conducted within one hour of treatment. Patients were asked about pain in the teeth, temporomandibular joint and muscles of mastication. The tongue, buccal and labial mucosae, teeth and restorations were examined for signs of trauma.

\section{Findings}

All patients admitted to hospital who were receiving ECT treatment or who had had ECT during their current admission were interviewed; only one patient was excluded because of severe psychotic symptoms. Thirty patients were interviewed, with a mean age of 47 years (range 20$82)$. There were seven males $(23 \%)$ and 23 females (77\%). Some form of depressive episode accounted for $93 \%$ of subjects, with one case of emotionally unstable personality disorder and one case of schizophrenia making up the total. Only one subject was totally medication free and 83\% were taking drugs with anticholinergic properties and capable of inducing xerostomia (dry mouth). Symptoms suggestive of temporomandibular joint dysfunction (pain and clicking) were found in $13(43 \%)$ subjects and four (15\%) complained of toothache. The most common symptom was xerostomia, occurring in 93\% of subjects. Drugs with anticholinergic properties were being taken by $86 \%$ of this group. Artificial means of keeping the mouth moist were utilised by two-thirds. Drinking sugary soft drinks and sucking boiled sweets were particularly popular, but only one patient used an artificial saliva spray obtained from a dentist.

Soft tissue abnormalities were found in 16 (53\%), including aphthous ulceration, geographic tongue. denture stomatitis, desquamative gingivitis and in a patient suffering from Crohns disease, lip swelling and moderate thickening of the oral mucosa was noted. The most prevalent occlusion type was class 2 , division I, present in $60 \%$.

The study population consisted of 19 (63\%) dentate, eight $(27 \%)$ partially dentate and three $(10 \%)$ edentulous patients. Three subjects were wearing partial upper dentures, one partial upper and full lower dentures. Four patients were wearing full upper dentures while having lower anterior teeth present. Two of the partially 
dentate patients required replacement or repair of their prostheses.

The mean number of sound teeth per dentate subject was 15 . The mean decayed, missing and filled teeth was 15 (1 decayed, 9 missing and 5 filled). The number of sound teeth declined significantly with age. At least one broken tooth was found in $10(37 \%)$ subjects.

Twenty subjects $(67 \%)$ claimed to brush their teeth at least twice a day. In contrast on examination oral hygiene was poor with 16 (56\%) having heavy interdental plaque accumulation. some with large deposits of calculus (plaque index 2 or 3 ). Fifteen (50\%) lacked regular dental care, visiting the dentist only if in pain. Financial difficulties and anxiety were the main reasons given for not having regular check-ups.

Twenty-six dentate patients were examined with the BPE probe (one patient was not examined due to a valvular heart defect). On probing. $24(92 \%)$ subjects had BPE scores of two or three, with bleeding at two sites occurring in $11(42 \%)$. In six $(23 \%)$ subjects the peridontal disease was so severe that some teeth were mobile and clearly a potential hazard during ECT.

A total of 68 treatments were observed and brief oral examinations performed. The most common complaint was of pain in the temporomandibular region following $44 \%$ of treatments. Minor soft tissue trauma occurred quite frequently, particularly in the posterior regions of the mouth (Table 1). The most serious complication was a fractured tooth.

\section{Comment}

Though the sample size was small it was representative of patients receiving treatment within a large teaching hospital serving an inner-city area. Compliance was good perhaps because an oral examination is a standard part of the pre-ECT assessment, but probably a more important factor was that the dental examination

Table 1. Symptoms and signs of oral trauma following ECT treatment ( $n=68)$

\begin{tabular}{ll}
\hline Symptom/sign & Frequency, \% \\
\hline Temporomandibular pain & 44 \\
Buccal lesion & 22 \\
Gingival lesion & 13 \\
Toothache & 13 \\
Labial lesion & 3 \\
Tongue lesion & 3 \\
Tooth fracture & 1.5 \\
Restoration fracture & 0 \\
Denture damage & 0 \\
\hline
\end{tabular}

was performed on a ward rather than in the dentist's chair.

Xerostomia was very common and often drug induced. Few patients could recall being given any advice on how to deal with a dry mouth and most were adopting maladaptive coping strategies. Prescription of artificial saliva sprays may be helpful if this symptom is severe.

The number of decayed missing and filled teeth seemed high, however 1988 figures for the mean decayed, missing and filled teeth in West Midlands adults was 16 ( 1 decayed, 7.6 missing and 7.4 filled), suggesting the study population to be slightly better than average. Xerostomia, poor oral hygiene, lack of dental guidance, a cariogenic diet and partial dentures may all be contributing to the high rate of caries. Poor oral hygiene is a major cause of peridontal disease and was present in over $90 \%$ of the sample. All patients needed improved oral hygiene, twothirds required scaling and cleaning and onethird complex peridontal therapy. A central problem seemed to be lack of dental education both for the patients and their carers, and despite the high rate of pathology none of the patients were registered with the hospital dentist.

Approximately one-half of the sample had partial dentures or fixed prostheses. Dentures replacing the anterior teeth should be removed, however strong prostheses replacing molars and premolars are often best left in place as they may stabilise dentition and balance the occlusion. Non-removable prostheses such as crowns and bridges should be assessed as to their suitability to withstand strong occlusal force, a bridge with a pontic area spanning more than three to four teeth may fail due to excessive strain on the restorations present on the natural teeth.

Pain in the temporomandibular region was the most common post-treatment complaint and may be due to joint strain. masticatory muscle fatigue or the effects of suxamethonium. Onefifth of patients sustained minor soft tissue trauma to the buccal mucosa. mainly in the posterior regions of the mouth. We felt that two factors may have contributed to this. First, the polystyrene disposable mouthguards were of one size and sometimes did not extend back sufficiently far into the mouth to cover the first and second molars and displace the buccal mucosa. Second, some anaesthetists failed to support the jaw, allowing possible displacement of the guard.

Opinion is divided as to whether all patients must have a pre-ECT examination by a dental practitioner (McClure, 1969; Weiner \& McCall, 1992). The high rate of relevant dental pathology would support specialist assessment, however resources are scarce and it is probably 
necessary to take a pragmatic approach with the medical staff screening patients and referring those thought to be particularly at risk.

We have now introduced clinical guidelines on dental management of patients receiving ECT (Appendix) and would recommend that similar guidelines are widely introduced.

\section{Appendix Guidelines on dental management for ECT}

1. Patients should be asked about relevant dental information, including the frequency of visits to the dentist, presence of dental restorations and prostheses, symptoms such as toothache, temporomandibular joint pain, loose teeth and bleeding gums.

2. Patients should undergo a brief oral examination with the aim of identifying:

(d) loose, broken, decayed or missing teeth, gross dental tartar, failed restorations and abnormal occlusions;

(e) removable dental prostheses (dentures and partial dentures);

(f) non-removable dental prostheses (cemented crowns, bridges and dental implants):

(g) soft tissue lesions and swellings:

(h) mandibular deformities;

(i) temporomandibular joint dysfunction (popping, clicking, crepitus and locking).

3. Patients with significant dental symptoms or abnormalities on examination (other than simply being edentulous) should be referred for an opinion from a dentist with knowledge and experience of the effects of ECT on oral structures.

4. Consideration should be given to dental risks during the consent procedure.

5. Information on the patients dental state should be made available to the ECT treatment team, preferably via the ECT treatment card.

6. A mouthguard should be used for all patients including the fully dentate and edentulous.

7. The mouthguard should be constructed of a material that is partly compressible, and extend sufficiently far back into the mouth to separate the first and second molar teeth. The guard should displace the tongue and buccal mucosa from opposing teeth or ridges and avoid overloading anterior dental structures. More than one size should be available.
8. The decision to retain partial dentures is dependent on the position. composition and stability of the prosthesis, the aim being to evenly distribute the load through structures with the ability to cope.

9. The jaws should be firmly held together by hand pressure on the mandible throughout the seizure in order to prevent the mouthguard from becoming displaced.

10. Attention should be paid to any abnormal sound coming from the mouth during the seizure. The mouthguard must be carefully removed and the oral cavity inspected for evidence of bleeding or loose and missing teeth. In recovery the patients should be asked about oral pain and difficulty in opening and closing the mouth. If significant abnormalities are identified these should be documented and the patient referred for a post-ECT dental evaluation.

\section{References}

AINAMO, J. BARNes, D. BEAGRe, G. et al (1982) Development of the World Health Organization community peridontal index of treatment needs. International Dental Joumal. 32. 281-291.

DURRANT. B. (1966) Dental care in electroplexy. British Journal of Psychiatry. 112. 1173-1176.

MCCLURE, R. E. (1969) A device for preventing dental injuries during ECT. Hospital and Community Psychiatry. 20. 357-359.

Minneman, S. A. (1995) A history of oral protection for the ECT patient: past, present and future. Convulsive Therapy. 11, 94-103.

ROYAL COLLEgE OF PSYCHIATRISTS (1995) The ECT Handbook. The Second Report of the Royal College of Psychiatrists Special Committee on ECT (Council Report CR39). London: Royal College of Psychiatrists.

SLAWSON. P. (1989) Psychiatric malpractice and ECT: a review of national loss experience. Convulsive Therapy. 5. 126-130.

WEINER, R. D. \& MCCALL, W. V. (1992) Dental consultation in ECT. Convulsive Therapy. 8, 146.

WORLD HEALTH ORGanization (1992) The Tenth Revision of the International Classification of Diseases and Related Health Problems (ICD-10). WHO: Geneva.

Nita Beli, Final Year Dental Student, Birmingham Dental Hospital and School, Birmingham; and *Peter Bentham, Consultant in Old Age Psychiatry. The Queen Elizabeth Psychiatric Hospital, Edgbaston, Birmingham B15 2QZ

*Correspondence 